
\title{
R Research Soure \\ Collocation Method for Fuzzy Volterra Integral Equation of the Second Kind with Weakly Singular Kernels
}

Zahra Alijani ( $\sim$ zahraalijani2@gmail.com )

University of Tartu: Tartu Ulikool

Urve Kangro

University of Tartu: Tartu Ulikool

\section{Research Article}

Keywords: Fuzzy Volterra, Singular Kernel

Posted Date: October 25th, 2021

DOI: https://doi.org/10.21203/rs.3.rs-393517/v1

License: (c) (1) This work is licensed under a Creative Commons Attribution 4.0 International License.

Read Full License 


\title{
COLLOCATION METHOD FOR FUZZY VOLTERRA INTEGRAL EQUATION OF THE SECOND KIND WITH WEAKLY SINGULAR KERNELS
}

\author{
ZAHRA ALIJANI $^{\dagger} *$ AND URVE KANGRO*
}

\begin{abstract}
In this paper, we consider fuzzy Volterra integral equation of the second kind with a weakly singular kernel. We propose piecewise spline collocation methods with a graded mesh. By increasing the number of collocation points we show that the numerical solution exists and converges to the exact solution. We obtain exact convergence values depending on the smoothness of the solution and on the grading parameter of the mesh. We give sufficient conditions for the fuzziness of the approximate solution. The proposed method is illustrated by numerical examples which confirm the theoretical convergence estimates.
\end{abstract}

\section{INTRODUCTION}

Integral equations and their solutions play a significant role in science and engineering. Many important physical problems can be modeled by using integral or differential equations. Only a few of them can be solved explicitly, so it is necessary to engage numerical methods to obtain approximate solutions [7].

The theory of Volterra integral equations is thoroughly considered by Brunner [6]. Weakly singular integral equations have received considerable interest in the mathematical literature, because of their applications in many fields of science such as fractional differential equations and the physical problems with heredity and memory properties [6, 19].

Usually, Volterra integral equations can not be solved analytically. Hence numerical methods for these equations are needed. The simplest and at the same time robust methods which are all to deal with the nonsmooth solution are collocation methods with piecewise polynomials [2, 7].

Many natural phenomena are described by mathematical models. It is not always possible to find a deterministic model for such problems. In 1965, Zadeh introduced the concept of fuzzy sets. With time, this concept found interesting applications in the modeling of many real-world problems and it motivated many researchers to develop the area of fuzzy integral equations where the values of functions involved are fuzzy numbers. The

\footnotetext{
* This work has been granted by the Estonian Research Council grant PRG864.
}

$\dagger$ corresponding. 
topics related to fuzzy integral equations have received particular attention from the research community during the last few decades [4, 10, 17, 18].

A fuzzy Volterra integral equation of the second kind with the weakly singular kernel (FVIEW) is given by

$$
g(t)=f(t)+\int_{0}^{t} K(t, s) g(s) d s, \quad t \in[0, T],
$$

where $K: D_{T} \rightarrow \mathbb{R}$ is a weakly singular kernel with domain $D_{T}=\{(t, s): 0 \leq$ $s<t \leq T\}, T \in \mathbb{R}, f$ is a given fuzzy function and $g$ is an unknown fuzzy function. The kernel $K$ or its derivatives may have some singularities at $t=s$. If $f$ is a real-valued function then equation (1.1) possesses a realvalued solution and if $f$ is a fuzzy-number valued function then the solution is fuzzy.

In our previous paper [3] we investigated fuzzy Volterra integral equations with weakly singular kernels. We obtained the existence and uniqueness of solutions and then we showed that the corresponding solution is a fuzzy function that satisfies equation (1.1). We proved the smoothness of the solution, assuming that the sign of the kernel can change only along the horizontal and vertical lines.

In current work we use fully discretized collocation methods on piecewise polynomial spaces for solving the corresponding system of Volterra integral equations. We provide the conditions for the fuzziness of the numerical solutions. Based on smoothness results we obtain the convergence analysis.

The paper is organized as follows. Section 2 introduces some preliminaries about fuzzy functions which are necessary for the next sections. In section 3 we describe the parametric and operator form of the integral equation. Section 4 consists of existence and uniqueness results for the parametric form. In Section 5 we describe the collocation method and fully discretized collocation method on the discontinuous piecewise polynomial spaces. In section 6 we prove the convergence results. Some examples are presented in section 7 to illustrate our result.

\section{PRELIMINARIES}

In this section, we review the fundamental notions and definitions that will be used throughout the paper.

2.1. Fuzzy numbers and fuzzy functions. Fuzzy sets $\mathbb{R}$ (generally on $\mathbb{R}^{n}, n \geq 1$ ) were first introduced in [21]. Fuzzy numbers are particular fuzzy sets, identified with some additional properties. Since we only use the parametric form of the fuzzy numbers in this paper, we only introduce this form. The parametric form was introduced in [12], where also the equivalence of the standard definition and the parametric form was proved.

Definition 2.1. A fuzzy number $u$ on $\mathbb{R}$ in parametric form is an ordered pair $u=(\underline{u}, \bar{u})$ of two real functions $\underline{u}, \bar{u}:[0,1] \rightarrow \mathbb{R}$, that satisfy the following requirements: 
(1) $\underline{u}$ is bounded, non-decreasing, left continuous on $(0,1]$ and right continuous at 0 ,

(2) $\bar{u}$ is bounded, non-increasing, left continuous on $(0,1]$ and right continuous at 0 ,

(3) $\underline{u}(1) \leq \bar{u}(1)$.

For arbitrary fuzzy numbers $u=(\underline{u}, \bar{u}), v=(\underline{v}, \bar{v})$ and $k \in \mathbb{R}$ the addition and multiplication by $k$ are defined as

$$
\begin{aligned}
& u+v=(\underline{u}+\underline{v}, \bar{u}+\bar{v}), \\
& k u= \begin{cases}(k \underline{u}, k \bar{u}), & \text { if } k \geq 0, \\
(k \bar{u}, k \underline{u}), & \text { if } k<0 .\end{cases}
\end{aligned}
$$

The set of all fuzzy numbers is denoted by $E$. Note that $E$ is not a vector space, because $u+(-u) \neq 0$ in general.

Next we will define the metric $D$ in $E$.

Definition 2.2. For arbitrary fuzzy numbers $u, v$, we use the distance

$$
D(u, v)=\sup _{0 \leq r \leq 1} \max \{|\bar{u}(r)-\bar{v}(r)|,|\underline{u}(r)-\underline{v}(r)|\} .
$$

It is shown that $(E, D)$ is a complete metric space [5].

Following Goetschel and Voxman [12] we define the integral of a fuzzy function using the Riemann integral concept.

Definition 2.3. Let $f:[a, b] \rightarrow E$. For each partition $P=\left\{t_{0}, \ldots, t_{n}\right\}$ of $[a, b]$ and for arbitrary $\xi_{i} \in\left[t_{i-1}, t_{i}\right], 1 \leq i \leq n$ suppose

$$
R_{P}=\sum_{i=1}^{n} f\left(\xi_{i}\right)\left(t_{i}-t_{i-1}\right), \quad \Delta:=\max \left\{t_{i}-t_{i-1}, i=1, \ldots, n\right\} .
$$

The definite integral of $f(t)$ over $[a, b]$ is $\int_{a}^{b} f(t) d t=\lim _{\Delta \rightarrow 0} R_{P}$ provided this limit exists in metric $D$.

If the fuzzy function $f(t)$ is continuous in the metric $\mathrm{D}$, its definite integral exists and

$$
\int_{a}^{b} f(t) d t=\left(\int_{a}^{b} \underline{f}(t, r) d t, \int_{a}^{b} \bar{f}(t, r) d t\right)
$$

where $(f(t, r), \bar{f}(t, r))$ is the parametric form of $f(t)$. In the literature, weak singularity of the kernel $K$ may have different definitions. We follow here the definition of [20], where it was introduced for Fredholm integral equations.

Definition 2.4. For given $m \in \mathbb{N}_{0}$, denote by $S^{m, \alpha}=S^{m, \alpha}\left(D_{T}\right)$ the set of $m$ times continuously differentiable functions $K$ on $D_{T}$ that satisfy there for all $j, l \in \mathbb{N}_{0}, j+l \leq m$, the inequality

$$
\left|\left(\frac{\partial}{\partial t}\right)^{j}\left(\frac{\partial}{\partial t}+\frac{\partial}{\partial s}\right)^{l} K(t, s)\right| \leq C_{K, m} \begin{cases}1 & \text { if } j+\alpha<0 \\ 1+|\log (t-s)| & \text { if } j+\alpha=0 \\ (t-s)^{-j-\alpha} & \text { if } j+\alpha>0 .\end{cases}
$$


A kernel $K \in S^{m, \alpha}$ is called weakly singular if $\alpha<1$.

To describe the smoothness of the solution of (1.1) we need the following space of functions.

Definition 2.5. [20] For $m \in \mathbb{N}_{0}, \alpha<1$, denote by $\mathcal{C}^{m, \alpha}(0, T]$, the space of functions $v \in \mathcal{C}^{m}(0, T]$, that satisfy the inequalities

$$
\left|v^{(i)}(t)\right| \leq c \begin{cases}1 & \text { if } i<1-\alpha \\ 1+|\log (t)| & \text { if } i=1-\alpha \\ t^{1-\alpha-i} & \text { if } i>1-\alpha\end{cases}
$$

where $c=c(v)$, for all $t \in(0, T]$ and $i=0, \ldots, m$.

For $\alpha \in \mathbb{R}$ we define the weight function

$$
\left|\omega_{\alpha}(t)\right|= \begin{cases}1 & \text { if } \quad \alpha<0 \\ (1+|\log (t)|)^{-1} & \text { if } \quad \alpha=0 \\ t^{\alpha} & \text { if } \quad \alpha>0 .\end{cases}
$$

Then $\mathcal{C}^{m, \alpha}(0, T]$, equipped with the norm

$$
\|v\|_{m, \alpha}:=\sum_{k=0}^{m} \sup _{0<t \leq T} \omega_{k-1+\alpha}(t)\left|v^{(k)}(t)\right|,
$$

becomes a Banach space and for $m \geq 1$.

\section{PARAmetric AND OPERATOR FORM OF THE INTEGRAL EQUATION}

Let $(f(t, r), \bar{f}(t, r))$ and $(g(t, r), \bar{g}(t, r)),(t, r) \in[0, T] \times[0,1]$ be parametric forms of $f(t)$ and $g(t)$. Then equation (1.1) can be rewritten as a system of Volterra integral equations:

$$
\left\{\begin{array}{l}
\underline{g}(t, r)=\underline{f}(t, r)+\int_{0}^{t}\left(K_{+}(t, s) \underline{g}(s, r)-K_{-}(t, s) \bar{g}(s, r) d s\right), \\
\bar{g}(t, r)=\bar{f}(t, r)+\int_{0}^{t}\left(K_{+}(t, s) \bar{g}(s, r)-K_{-}(t, s) \underline{g}(s, r) d s\right)
\end{array}\right.
$$

where

$$
K_{+}(t, s)= \begin{cases}K(t, s), & K(t, s) \geq 0 \\ 0, & \text { otherwise }\end{cases}
$$

and

$$
K_{-}(t, s)= \begin{cases}-K(t, s), & K(t, s) \leq 0 \\ 0, & \text { otherwise }\end{cases}
$$

We write this system as

$$
G=F+\mathcal{K} G,
$$

where $G=\left[g_{1}, g_{2}\right]^{T}, g_{1}=\underline{g}, g_{2}=\bar{g}, F=\left[f_{1}, f_{2}\right]^{T}, f_{1}=\underline{f}, f_{2}=\bar{f}$ and

$$
\mathcal{K}=\left(\begin{array}{cc}
\mathcal{K}_{\alpha_{+}} & -\mathcal{K}_{\alpha_{-}} \\
-\mathcal{K}_{\alpha_{-}} & \mathcal{K}_{\alpha_{+}}
\end{array}\right)
$$


We also use the notation

$$
\mathcal{K} G=\int_{0}^{t} \mathbf{K}(t, s) G(s, r) d s,
$$

where

$$
\mathbf{K}(t, s)=\left(\begin{array}{cc}
K_{+}(t, s) & -K_{-}(t, s) \\
-K_{-}(t, s) & K_{+}(t, s)
\end{array}\right) .
$$

We call the vector $G$ a fuzzy function if $\left(g_{1}, g_{2}\right)$ is a fuzzy function.

In [3] we proved the existence, uniqueness and smoothness of the solution. Here we review them.

\section{Existence, Uniqueness AND SMOOTHNess of THE SOLUTION}

In [3] we suppose the kernel changes sign along the vertical and/or horizontal lines $s=a_{i}$ and/or $t=a_{i}, i=1, \ldots, n, 0<a_{1}<a_{2}<\ldots<a_{n}<T$. Denote

$$
D_{\left\{a_{1}, \ldots, a_{n}\right\}}=D_{T} \backslash \bigcup_{i=1}^{n}\left(\left\{s=a_{i}\right\} \cup\left\{t=a_{i}\right\}\right) .
$$

Define $S^{m, \alpha}\left(D_{\left\{a_{1}, \ldots, a_{n}\right\}}\right)$ as the collection of $m$ times continuously differentiable functions $K$ on $D_{\left\{a_{1}, \ldots, a_{n}\right\}}$ that satisfy inequality (2.2) for all $j, l \in \mathbb{N}_{0}$, $j+l \leq m$ and $(t, s) \in D_{\left\{a_{1}, \ldots, a_{n}\right\}}$. Without loss of generality we assumed there is only one vertical and/or horizontal line of sign change of $K$. Denote $d=a_{1}$ and $D_{d}=D_{T} \backslash(\{s=d\} \cup\{t=d\})$. In order to characterize the growth rates of the derivatives of the function $u(t)$ as $t \rightarrow d$, Pedas et al. [15] introduced also the weight functions

$$
\left|\omega_{\alpha}^{(d)}(t)\right|= \begin{cases}1 & \text { if } \alpha<0 \\ \left(1+\left|\log \left(\rho_{d}\right)\right|\right)^{-1} & \text { if } \alpha=0 \\ \rho_{d}(t)^{\alpha} & \text { if } \alpha>0\end{cases}
$$

where $t \in \mathcal{G}_{d}$ and $\rho_{d}=|t-d|$.

We denote by $\mathcal{C}_{d}^{m, \alpha, p}(0, T]$ the Banach space of functions $u \in \mathcal{C}^{m}((0, T] \backslash$ $\{d\}) \cap \mathcal{C}^{p}(0, T]$ such that

$$
\begin{gathered}
\|u\|_{d, m, \alpha, p}=\sum_{j=0}^{m} \sup _{t \in \mathcal{G}_{d}} e(t) \omega_{j+\alpha-1}(t)\left|u^{(j)}(t)\right|+ \\
\sum_{j=0}^{m} \sup _{t \in \mathcal{G}_{d}}(1-e(t)) \omega_{j+\alpha-1-p}(t-d)\left|u^{(j)}(t)\right|<\infty,
\end{gathered}
$$

where $\omega$ is defined in (2.4).

In [3] we provided some results for the case when the lines of sign change can only be vertical and/or horizontal lines.

Theorem 4.1. Let $K_{+}, K_{-} \in S^{m, \alpha}\left(D_{d}\right) \cap \mathcal{C}^{p-1}\left(D_{T}\right)$ where $m, p \in \mathbb{N}, p \leq$ $m, \alpha \in \mathbb{R}, \alpha<1$. Let $F \in\left(\mathcal{C}_{d}^{m, \alpha, p}(0, T]\right)^{2}$ be given. Then the equation (3.2) has a unique solution in $\left(\mathcal{C}_{d}^{m, \alpha, p}(0, T]\right)^{2}$. 


\section{Collocation methods}

\subsection{Collocation method on the discontinuous piecewise polyno-} mial spaces. Define a mesh on $[0, T]$ by

$$
\triangle_{h}:=\left\{t_{n}: 0=t_{0}<t_{1}<\ldots<t_{N}=T\right\} .
$$

Let $\sigma_{n}:=\left(t_{n}, t_{n+1}\right], \bar{\sigma}_{n}:=\left[t_{n}, t_{n+1}\right], h_{n}=t_{n+1}-t_{n}(n=0,1, \ldots, N-1)$ and let the diameter of the mesh be $h=\max \left\{h_{n}: 0 \leq n \leq N-1\right\}$. In the following we mainly use the graded mesh, where the mesh points are defined by

$$
t_{j}:=T\left(\frac{j}{N}\right)^{\rho}, \quad j=0, \ldots, N .
$$

Here $\rho \geq 1$ is called the grading parameter.

Define the piecewise polynomial space which we use in this thesis as follows:

$$
\mathcal{S}_{m-1}^{(-1)}\left(\triangle_{h}\right):=\left\{v:\left.v\right|_{\sigma_{n}} \in \pi_{m-1}(n=0,1, \ldots, N-1)\right\},
$$

where $\pi_{m-1}$ are polynomials of degree not exceeding $m-1$. Any $v_{N} \in$ $\left(\mathcal{S}_{m-1}^{(-1)}\left(\triangle_{h}\right)\right)$ can be determined by

$$
\left.v_{N}(t)\right|_{\sigma_{n}}=\sum_{j=1}^{m} L_{j}(\tau) V_{n, j}, \quad t=t_{n}+\tau h_{n},
$$

where $V_{n, i}:=v_{N}\left(t_{n, i}\right), 0 \leq c_{1}<\ldots<c_{m} \leq 1$ are the collocation parameters, $t_{n, i}:=t_{n}+c_{i} h_{n}$ for $n=0, \cdots, N-1$, and

$$
L_{j}(\tau):=\prod_{k=1, \ldots, m, k \neq j} \frac{\tau-c_{k}}{c_{j}-c_{k}}, j=1, \ldots, m, \tau \in[0,1],
$$

are the Lagrange fundamental polynomials on $[0,1]$.

For fixed $r \in[0,1]$ we look for approximate solution of equation (3.2) as a spline $u_{N} \in\left(\mathcal{S}_{m-1}^{(-1)}\left(\triangle_{h}\right)\right)^{2}$. We require that the equation is exactly satisfied at collocation points $t_{n, i}$. Then we get the linear system of equations

$$
u_{N}\left(t_{n, i}, r\right)=F\left(t_{n, i}, r\right)+\left(\mathcal{K} u_{N}\right)\left(t_{n, i}, r\right) .
$$

for determining $u_{N}\left(t_{n, i}, r\right)$. By partitioning the integration interval in equation (3.2) we obtain

$$
\begin{aligned}
u_{N}\left(t_{n, i}, r\right)= & F\left(t_{n, i}, r\right)+\sum_{l=0}^{n-1} h_{l} \int_{0}^{1} \mathbf{K}\left(t_{n, i}, t_{l}+z h_{l}\right) u_{N}\left(t_{l}+z h_{l}, r\right) d z \\
& +h_{n} \int_{0}^{c_{i}} \mathbf{K}\left(t_{n, i}, t_{n}+z h_{n}\right) u_{N}\left(t_{n}+z h_{n}, r\right) d z
\end{aligned}
$$

Denote $U_{n, i}(r)=u_{N}\left(t_{n, i}, r\right)$. Note that we can solve the equations on each interval $\sigma_{n}$ separately, so when solving for $U_{n, i}(r)$ for fixed $n$, we can consider 
$U_{l, j}$ with $l<n$ as known. By substituting (5.2) into (5.4), we obtain

$$
\begin{aligned}
U_{n, i}(r)= & h_{n} \sum_{j=1}^{m} \int_{0}^{c_{i}} \mathbf{K}\left(t_{n, i}, t_{n}+z h_{n}\right) L_{j}(z) d z U_{n, j}(r) \\
& +F\left(t_{n, i}, r\right)+\sum_{l=0}^{n-1} \sum_{j=1}^{m} h_{l} \int_{0}^{1} \mathbf{K}\left(t_{n, i}, t_{l}+z h_{l}\right) L_{j}(z) d z U_{l, j}(r) .
\end{aligned}
$$

For fixed $n$ we obtain a linear system of $2 m$ equations and $2 m$ unknowns

$$
\begin{aligned}
U_{n, i}(r)= & h_{n} \sum_{j=1}^{m} Q_{n, i, j} U_{n, j}(r) \\
& +F\left(t_{n, i}, r\right)+\sum_{l=0}^{n-1} \sum_{j=1}^{m} h_{l} R_{n, l, i, j} U_{l, j}(r), \quad i=1, \ldots, m,
\end{aligned}
$$

where

$$
Q_{n, i, j}=\int_{0}^{c_{i}} \mathbf{K}\left(t_{n, i}, t_{n}+z h_{n}\right) L_{j}(z) d z
$$

and

$$
R_{n, l, i, j}=\int_{0}^{1} \mathbf{K}\left(t_{n, i}, t_{l}+z h_{l}\right) L_{j}(z) d z .
$$

5.2. The fully discretized collocation method. To describe the fully discretized collocation method we make an additional assumption:

$$
K(t, s)=k(t, s) p_{\alpha}(t-s), \quad p_{\alpha}(t)= \begin{cases}t^{-\alpha}, & \alpha<1, \quad \alpha \neq 0, \\ \log (t), & \alpha=0,\end{cases}
$$

where $k \in \mathcal{C}^{m}\left(\overline{D_{T}}\right)$ and $\alpha<1$. Then $K \in S^{m, \alpha}$. Assume that the lines of sign change of $K$ are only at horizontal and/or vertical lines $t=t_{j}$ or $s=t_{j}$ for some $j \in\{1, \ldots, N-1\}$. Then all integrals in (5.4) are either zero or of the form

$$
\int_{0}^{a} k\left(t_{n, i}, t_{l}+z h_{l}\right) p_{\alpha}\left(t_{n, i}-\left(t_{l}+z h_{l}\right)\right) v\left(t_{l}+z h_{l}\right) d z
$$

with $a=1$ or $a=c_{i}$. We approximate these integrals by product quadrature rule with the mesh $\left\{c_{1}, \ldots, c_{m}\right\}$ as follows

$$
\int_{0}^{c_{i}} k\left(t_{n, i}, t_{n}+z h_{n}\right) p_{\alpha}\left(t_{n, i}-\left(t_{n}+z h_{n}\right)\right) v\left(t_{n}+z h_{n}\right) d z \approx \sum_{j=1}^{m} w_{n, i, j} k\left(t_{n, i}, t_{n}+c_{j} h_{n}\right) V_{n, j}
$$

and

$$
\int_{0}^{1} k\left(t_{n, i}, t_{l}+z h_{l}\right) p_{\alpha}\left(t_{n, i}-\left(t_{l}+z h_{l}\right)\right) v\left(t_{l}+z h_{l}\right) d z \approx \sum_{j=1}^{m} w_{n, l, i, j} k\left(t_{n, i}, t_{l}+c_{j} h_{l}\right) V_{l, j},
$$

where

$$
w_{n, i, j}:=\int_{0}^{c_{i}} p_{\alpha}\left(\left(c_{i}-z\right) h_{n}\right) L_{j}(z) d z
$$


and

$$
w_{n, l, i, j}:=\int_{0}^{1} p_{\alpha}\left(t_{n, i}-\left(t_{l}+z h_{l}\right)\right) L_{j}(z) d z .
$$

For fixed $r$ we look for approximate solution of equation (3.2) as a spline $\widehat{u}_{N}(\cdot, r) \in\left(\mathcal{S}_{m-1}^{(-1)}\left(\triangle_{h}\right)\right)^{2}$ and denote $\widehat{U}_{n, i}(r)=\widehat{u}_{N}\left(t_{n, i}, r\right)$. Then for determining $\widehat{U}_{n, i}(r)$ we get the following linear system of equations

$$
\begin{aligned}
\widehat{U}_{n, i}(r)= & h_{n} \sum_{j=1}^{m} \widehat{Q}_{n, i, j} \widehat{U}_{n, j}(r) \\
& +F\left(t_{n}+c_{i} h_{n}, r\right)+\sum_{l=0}^{n-1} \sum_{j=1}^{m} h_{l} \widehat{R}_{n, l, i, j} \widehat{U}_{l, j}(r), \quad i=1, \ldots, m,
\end{aligned}
$$

where

$$
\widehat{Q}_{n, i, j}=\mathbf{k}\left(t_{n}+c_{i} h_{n}, t_{n}+c_{j} h_{n}\right) w_{n, i, j}
$$

and

$$
\widehat{R}_{n, l, i, j}=\mathbf{k}\left(t_{n}+c_{i} h_{n}, t_{l}+c_{j} h_{l}\right) w_{n, l, i, j},
$$

where

$$
\mathbf{k}(t, s)=\left(\begin{array}{cc}
k(t, s) & 0 \\
0 & k(t, s)
\end{array}\right) \quad \text { or } \quad \mathbf{k}(t, s)=\left(\begin{array}{cc}
0 & -k(t, s) \\
-k(t, s) & 0
\end{array}\right),
$$

depending on whether $K(t, s)$ is positive or negative in $\sigma_{n} \times \sigma_{l}$. Then the approximate solution can be written as

$$
\left.\widehat{u}_{N}(t, r)\right|_{\sigma_{n}}=\sum_{j=1}^{m} L_{j}(z) \widehat{U}_{n, j}(r), \quad t=t_{n}+z h_{n}, \quad z \in(0,1] .
$$

\section{Convergence}

6.1. Convergence estimates for the collocation method. We denote by $p_{N}$ the interpolation projector onto the set of all piecewise polynomial functions on $[0, T]$ which are real polynomials of degree not exceeding $m-1$ on every interval $\left[t_{j}, t_{j+1}\right], 0 \leq j \leq N-1$, where the interpolation points are defined by $t_{n, i}=t_{n}+c_{i} h_{n}, 0 \leq c_{1}<\cdots<c_{m} \leq 1$. The approximation properties of $p_{N} u$ on graded mesh (5.1) are considered in [13, 16, 19]. These results can be summarized as follows.

Lemma 6.1. [16] Assume that $u \in \mathcal{C}^{m, \alpha}(0, T]$ and the graded mesh (5.1) with grading parameter $\rho$ is used. Then the following estimates hold where the constant $C$ does not depend on $N$ :

$$
\max _{t \in[0, T]}\left|u(t)-\left(p_{N} u\right)(t)\right| \leq C\|u\|_{m, \alpha} E(N, m, \rho, \alpha),
$$


where

(6.2)

$$
E(N, m, \rho, \alpha)= \begin{cases}N^{-m}, & \text { for } m<1-\alpha, \rho \geq 1, \\ N^{-m}(|\log N|+1), & \text { for } m=1-\alpha \rho=1, \\ N^{-m}, & \text { for } m=1-\alpha, \rho>1, \\ N^{-\rho(1-\alpha)}, & \text { for } m>1-\alpha, 1 \leq \rho<\frac{m}{1-\alpha}, \\ N^{-m}, & \text { for } m>1-\alpha, \rho \geq \frac{m}{1-\alpha} .\end{cases}
$$

Remark 6.2. If $u \in \mathcal{C}_{d}^{m, \alpha, p}(0, T]$, then we can use different graded meshes on $[0, d]$ and $[d, T]$, possibly with different grading parameters, and use Lemma 6.1 separately on these intervals.

In the consequent theorems we present the convergence result for fuzzy weakly singular integral equation.

Define an interpolation projector

$$
\mathbf{p}_{N}:(\mathcal{C}[0, T])^{2} \mapsto\left(\mathcal{S}_{m-1}^{(-1)}\left(\triangle_{h}\right)\right)^{2}, \quad m, N \in \mathbb{N},
$$

by

$$
\left(\mathbf{p}_{N} v\right)\left(t_{n, i}\right)=v\left(t_{n, i}\right), \quad i=1, \ldots, m, \quad n=0, \ldots, N,
$$

for any continuous function $v \in(\mathcal{C}(0, T])^{2}$.

Let $r \in[0,1]$ be fixed. Then the system (5.6) can be replaced by an operator equation of the form

$$
u_{N}(t, r)=\mathbf{p}_{N} F(t, r)+\mathbf{p}_{N} \mathcal{K} u_{N}(t, r) .
$$

Proposition 6.3. Assume that $F(., r) \in\left(\mathcal{C}^{m, \alpha}(0, T]\right)^{2}$ for all $r \in[0,1]$ and $\|F(\cdot, r)\|_{m, \alpha}$ are uniformly bounded with respect to $r$. Let $K_{+}, K_{-} \in S^{m, \alpha}$ with $m \geq 1, \alpha<1$. Let $G$ be the unique solution of the system (3.2). Let the graded mesh (5.1) with grading parameter $\rho$ be used. Then

$$
\sup _{r \in[0,1]}\left\|G(\cdot, r)-\mathbf{p}_{N} G(\cdot, r)\right\|_{\infty} \leq \operatorname{const} E(N, m, \rho, \alpha) .
$$

Proof. By Theorem 4.6 in [3] the operator $(I-\mathcal{K})$ is invertible in $\left(\mathcal{C}^{m, \alpha}(0, T]\right)^{2}$. Since $F(., r) \in\left(\mathcal{C}^{m, \alpha}(0, T]\right)^{2}$, we get $\|G(., r)\|_{m, \alpha} \leq\left\|(I-\mathcal{K})^{-1}\right\|\|F(., r)\|_{m, \alpha}$. Then by Lemma 6.1 we get (6.5).

In general case when the kernel $K$ changes sign, the assumptions of the previous proposition may be too restrictive. Then we have the following result.

Proposition 6.4. Assume that $F(., r) \in\left(\mathcal{C}^{m, \alpha}(0, T]\right)^{2}$ for all $r \in[0,1]$ and $\|F(\cdot, r)\|_{m, \alpha}$ are uniformly bounded with respect to $r$. Let $K_{+}, K_{-} \in$ $S^{m, \alpha}\left(D_{d}\right) \cap \mathcal{C}^{p-1}\left(D_{T}\right)$ with $m \geq 1, \alpha<1,1 \leq p \leq m$. Let $G$ be the unique solution of the system (3.2). Let two graded meshes on $[0, d]$ and on $[d, T]$ 
with numbers of intervals $N_{1}$ and $N_{2}$ and grading parameters $\rho_{1}$ and $\rho_{2}$ be used. Then

$$
\sup _{r \in[0,1]}\left\|G(\cdot, r)-\mathbf{p}_{N} G(\cdot, r)\right\|_{\infty} \leq \text { const } \max \left\{E\left(N_{1}, m, \rho_{1}, \alpha\right), E\left(N_{1}, m, \rho_{2}, \alpha-p\right)\right\} .
$$

Proof. The proof is similar to the proof of the previous proposition, only we use Theorem 4.9 in [3] and the space is $\left(\mathcal{C}_{d}^{m, \alpha, p}(0, T]\right)^{2}$.

Remark 6.5. Similar results hold when there are more lines of sign change of $K$.

We use the following general theorem about interpolation operator $p_{N}$.

Lemma 6.6. [8] Let $\mathcal{T}: \mathcal{L}^{\infty}(0, T) \rightarrow \mathcal{C}[0, T]$ be a linear compact operator. Let $p_{N}: \mathcal{C}[0, T] \rightarrow \mathcal{S}_{m-1}^{(-1)}\left(\triangle_{h}\right)$ be the interpolation operator with graded mesh (5.1). Then

$$
\left.\left\|\mathcal{T}-p_{N} \mathcal{T}\right\|_{\mathcal{L}(\mathcal{L} \infty(0, T), \mathcal{L}}{ }^{\infty}(0, T)\right) \rightarrow 0 \text {, as } N \rightarrow \infty .
$$

To establish convergence order we can use following theorem.

Theorem 6.7. Let $X, X^{\prime}$ be Banach spaces and $X^{\prime} \subset X$. Assume $\mathcal{T}: X \rightarrow$ $X^{\prime}$ is bounded and $I-\mathcal{T}: X \rightarrow X$, is bijective operator. Further, assume

$$
\left\|\mathcal{T}-p_{N} \mathcal{T}\right\| \rightarrow 0 \text {, as } N \rightarrow \infty,
$$

where $p_{N}: X^{\prime} \rightarrow X, N=1,2, \ldots$ are bounded linear operators. Then for all sufficiently large $N$ (say $N>N_{0}$ ) the operator $\left(I-p_{N} \mathcal{T}\right)$ is invertible in $X$ and

$$
\sup _{N>N_{0}}\left\|\left(I-p_{N} \mathcal{T}\right)^{-1}\right\|<\infty .
$$

For the solutions of $x_{N}=p_{N} \mathcal{T} x_{N}+p_{N} f$ and $x=\mathcal{T} x+f$,

$$
c_{1}\left\|x-p_{N} x\right\| \leq\left\|x-x_{N}\right\| \leq c_{2}\left\|x-p_{N} x\right\|,
$$

where $c_{1}$ and $c_{2}$ are positive constants.

Proof. This theorem is a generalization of Theorem 12.1.2 in [1] and the proof is similar.

Theorem 6.8. Assume that $F(., r) \in\left(\mathcal{C}^{m, \alpha}(0, T]\right)^{2}$ for all $r \in[0,1]$ and $\|F(\cdot, r)\|_{m, \alpha}$ are uniformly bounded with respect to $r$. Assume $K_{+}, K_{-} \in$ $\mathcal{S}^{m, \alpha}$. Let $G$ be the unique solution of the system (3.2). Assume that the collocation method (6.4) with collocation parameters $0 \leq c_{1}<\ldots<c_{m} \leq 1$, $m \in \mathbb{N}$ and with grading parameter $\rho \geq 1$ are used. Then there exists an integer $N_{0}$ such that for all $N \geq N_{0}$, operator equation (6.4) possesses a unique solution $u_{N}(., r) \in\left(\mathcal{S}_{m-1}^{(-1)}\left(\triangle_{h}\right)\right)^{2}$ and

$$
\sup _{r \in[0,1]}\left\|G(., r)-u_{N}(., r)\right\|_{\infty} \rightarrow 0, \quad N \rightarrow \infty .
$$


Furthermore, for $N \geq N_{0}$ the following error estimates hold:

$$
\sup _{r \in[0,1]}\left\|G(., r)-u_{N}(., r)\right\|_{\infty} \leq \operatorname{const} E(N, m, \rho, \alpha) .
$$

Proof. The conditions of Theorem 6.7 are satisfied with $X=\left(\mathcal{L}^{\infty}(0, T)\right)^{2}$, $X^{\prime}=(\mathcal{C}[0, T])^{2}, \mathcal{T}=\mathcal{K}, x=G(., r), x_{N}=u_{N}$ and $p_{N}=\mathbf{p}_{N}$. Thus

$$
\left\|G(., r)-u_{N}(., r)\right\|_{\infty} \leq c\left\|G(., r)-\mathbf{p}_{N} G(., r)\right\|_{\infty} .
$$

Now Proposition 6.3 completes the proof.

We state separately the case when the kernel $K$ changes sign.

Theorem 6.9. Assume that $F(., r) \in\left(\mathcal{C}^{m, \alpha}(0, T]\right)^{2}$ for all $r \in[0,1]$ and $\|F(\cdot, r)\|_{m, \alpha}$ are uniformly bounded with respect to $r$. Let $K_{+}, K_{-} \in S^{m, \alpha}\left(D_{d}\right) \cap$ $\mathcal{C}^{p-1}\left(D_{T}\right)$ with $m \geq 1, \alpha<1,1 \leq p \leq m$. Let $G$ be the unique solution of the system (3.2). Let the collocation method (6.4) with collocation parameters $0 \leq c_{1}<\ldots<c_{m} \leq 1, m \in \mathbb{N}$ and with two graded meshes on $[0, d]$ and on $[d, T]$ with numbers of intervals $N_{1}$ and $N_{2}$ and grading parameters $\rho_{1}$ and $\rho_{2}$ be used. Then there exists an integer $N_{0}$ such that for all $N \geq N_{0}$, operator equation (6.4) possesses a unique solution $u_{N}(., r) \in\left(\mathcal{S}_{m-1}^{(-1)}\left(\triangle_{h}\right)\right)^{2}$ and

$$
\sup _{r \in[0,1]}\left\|G(., r)-u_{N}(., r)\right\|_{\infty} \rightarrow 0, \quad N \rightarrow \infty .
$$

Furthermore, for $N \geq N_{0}$ the following error estimates hold:

$$
\sup _{r \in[0,1]}\left\|G(\cdot, r)-u_{N}(., r)\right\|_{\infty} \leq \text { const } \max \left\{E\left(N_{1}, m, \rho_{1}, \alpha\right), E\left(N_{1}, m, \rho_{2}, \alpha-p\right)\right\} .
$$

6.2. Convergence estimates for the fully discretized collocation method.

In deriving the fully dicretized collocation method, we approximated the integrals by the product quadrature rule. The quadrature rule was obtained by substituting the smooth part under the integral sign by its interpolation polynomial. The next lemma estimates the error of quadrature rule.

Lemma 6.10. Let $p_{\alpha}$ be defined as in (5.9). Let $p_{N}$ be the interpolation projector to spline space $S_{m-1}^{-1}\left(\Delta_{N}\right)$. Then the following estimates hold.

i) If $f \in \mathcal{C}^{m}[0, T]$ then

$$
\left|\int_{0}^{t} f(s) p_{\alpha}(s) d s-\int_{0}^{t}\left(p_{N} f\right)(s) p_{\alpha}(s) d s\right| \leq C \max \left|f^{(m)}(s)\right| h^{m} .
$$

ii) If $f \in C^{m, \alpha}(0, T]$ and the graded mesh with the grading parameter $\rho$ is used, then

$$
\left|\int_{0}^{t} f(s) p_{\alpha}(s) d s-\int_{0}^{t}\left(p_{N} f\right)(s) p_{\alpha}(s) d s\right| \leq C E(N, m, \rho, \alpha) .
$$

iii) If $k \in \mathcal{C}^{m}\left(\overline{D_{T}}\right)$ and $v_{N}(s) \in S_{m-1}^{-1}\left(\Delta_{h}\right)$, then

$$
\left|\int_{0}^{t} p_{\alpha}(t, s)\left[k(t, s) v_{N}(s)-p_{N}(k v)(t, s) d s\right]\right| \leq C h\|v\|_{\infty} .
$$


Proof. We use the standard estimate of the interpolation error:

$$
\left\|f-p_{N} f\right\|_{\infty} \leq C \max _{s \in[0, t]}\left|f^{(m)}(s)\right| h^{m}
$$

Then

$$
\begin{aligned}
\left|\int_{0}^{t} f(s) p_{\alpha}(s) d s-\int_{0}^{t}\left(p_{N} f\right)(s) p_{\alpha}(s) d s\right| & \leq \int_{0}^{t} p_{\alpha}(s) d s\left\|f-p_{N} f\right\|_{\infty} \\
& \leq C \max _{s \in[0, t]}\left|f^{(m)}(s)\right| h^{m} .
\end{aligned}
$$

This proves the first estimate. Proof of the second estimate is similar, using Lemma 6.1.

For the proof of assertion (iii) we start as before

$$
\begin{aligned}
& \left|\int_{0}^{t} p_{\alpha}(t, s)\left[k(t, s) v_{N}(s)-p_{N}(k v)(t, s)\right] d s\right| \\
& \leq \int_{0}^{t} p_{\alpha}(t-s) d s \max _{s \in[0, t]}\left|k(t, s) v_{N}(s)-p_{N}(k v)(t, s)\right|
\end{aligned}
$$

We estimate the interpolation error in each subinterval $\left[t_{l}, t_{l+1}\right]$ separately. Since $v_{N}$ is a polynomial of order $m-1$ in each subinterval, we have

$$
\max _{s \in\left[t_{l}, t_{l+1}\right]}\left|v_{N}^{(j)}(s)\right| \leq C h_{l}^{-j}\|v\|_{\infty}, \quad j=0,1, \ldots, m-1
$$

and $v_{N}^{(m)}(s)=0$. Hence we can estimate

$$
\begin{aligned}
& \max _{s \in\left[t_{l}, t_{l+1}\right]}\left|k\left(t_{n, i}, s\right) v_{N}(s)-p_{N}(k v)(t, s)\right| \\
\leq & C \max _{s \in\left[t_{l}, t_{l+1}\right]}\left|\frac{\partial^{m}}{\partial s^{m}}\left(k(t, s) v_{N}(s)\right)\right| h_{l}^{m} \leq C h_{l}^{-m+1} \cdot h_{l}^{m} \leq C h_{l} \leq C h .
\end{aligned}
$$

Now using the fact that $p_{\alpha}$ is integrable, (6.12) and (6.13) give the desired estimate.

To prove the convergence of the fully discretized collocation method we use the following general theorem about convergence of projection methods, when the operator is first approximated. It is similar to Corollary 13.11 of [14], but since one of the assumptions is not satisfied in our case, we give a new proof. Similar results have also been proved in [7], but our results are more general.

Theorem 6.11. Let $X, X^{\prime}$ be Banach spaces and $X^{\prime} \subset X$. Assume $\mathcal{T}: X \rightarrow$ $X^{\prime}$ is bounded and $I-\mathcal{T}: X \rightarrow X$ is injective operator. Assume

$$
\left\|\mathcal{T}-p_{N} \mathcal{T}\right\| \rightarrow 0, \text { as } N \rightarrow \infty
$$


where $p_{N}: X^{\prime} \rightarrow X, N=1,2, \ldots$ are bounded linear operators. Let $X_{N}=$ $p_{N}\left(X^{\prime}\right)$. Let $\mathcal{T}_{N}: X_{N} \rightarrow X$ be an approximation of $\mathcal{T}$ such that

$$
\sup _{v_{N} \in X_{N},\left\|v_{N}\right\|=1}\left\|\left(p_{N} \mathcal{T}_{N}-p_{N} \mathcal{T}\right) v_{N}\right\| \rightarrow 0, \quad N \rightarrow \infty .
$$

Then for all sufficiently large $N$ the operator $I-p_{N} \mathcal{T}_{N}$ is invertible in $X_{N}$ and

$$
\sup _{N>N_{0}}\left\|\left(I-p_{N} \mathcal{T}_{N}\right)^{-1}\right\|_{X_{N}}<\infty .
$$

For the solutions of $\widehat{x}_{N}=p_{N} \mathcal{T}_{N} \widehat{x}_{N}+p_{N} f_{N}$ and $x=\mathcal{T} x+f$ we have the estimate

$$
\left\|x-\widehat{x}_{N}\right\| \leq C\left(\left\|x-p_{N} x\right\|+\left\|p_{N}\left(\mathcal{T}_{N} p_{N}-\mathcal{T}\right) x\right\|+\left\|p_{N}\left(f_{N}-f\right)\right\|\right),
$$

where $C$ is a positive constant.

Proof. Since $I-p_{N} \mathcal{T}_{N}=\left(I-p_{N} \mathcal{T}\right)+\left(p_{N} \mathcal{T}-p_{N} \mathcal{T}_{N}\right), I-p_{N} \mathcal{T}$ is invertible in $X_{N}$ (see Theorem 6.7) and $\left\|p_{N} \mathcal{T}_{N}-p_{N} \mathcal{T}\right\|_{X_{N}} \rightarrow 0$, invertibility of $I-p_{N} \mathcal{T}_{N}$ in $X_{N}$ and uniform boundedness of the inverse operators follows.

Let $x_{N}$ be the solution of $x_{N}=p_{N} \mathcal{T} x+p_{N} f$ (note that the assumptions of Theorem 6.7 are satisfied). Subtracting the equations for $x_{N}$ and $\widehat{x}_{N}$ we get

$$
x_{N}-\widehat{x}_{N}=p_{N} \mathcal{T}_{N}\left(x_{N}-\widehat{x}_{N}\right)-\left(p_{N} \mathcal{T}_{N}-p_{N} \mathcal{T}\right) x_{N}+p_{N} f-p_{N} f_{N},
$$

hence

$$
\left\|x_{N}-\widehat{x}_{N}\right\| \leq\left\|\left(I-p_{N} \mathcal{T}_{N}\right)^{-1}\right\|\left(\left\|\left(p_{N} \mathcal{T}_{N}-p_{N} \mathcal{T}\right) x_{N}\right\|+\left\|p_{N} f-p_{N} f_{N}\right\|\right) .
$$

We can estimate $\left\|\left(p_{N} \mathcal{T}_{N}-p_{N} \mathcal{T}\right) x_{N}\right\|$ as follows, using Theorem 6.7:

$$
\begin{aligned}
& \left\|\left(p_{N} \mathcal{T}_{N}-p_{N} \mathcal{T}\right) x_{N}\right\| \leq\left\|\left(p_{N} \mathcal{T}_{N}-p_{N} \mathcal{T}\right)\left(x_{N}-p_{N} x\right)\right\| \\
& \quad+\left\|p_{N} \mathcal{T}\left(x-p_{N} x\right)\right\|+\left\|\left(p_{N} \mathcal{T}_{N} p_{N}-p_{N} \mathcal{T}\right) x\right\| \\
& \leq\left\|p_{N} \mathcal{T}_{N}-p_{N} \mathcal{T}\right\|_{X_{N}}\left\|x_{N}-p_{N} x\right\|+\left\|p_{N} \mathcal{T}\right\|\left\|x-p_{N} x\right\|+\left\|\left(p_{N} \mathcal{T}_{N} p_{N}-p_{N} \mathcal{T}\right) x\right\| \\
& \quad \leq C\left\|p_{N} x-x\right\|+\left\|\left(p_{N} \mathcal{T}_{N} p_{N}-p_{N} \mathcal{T}\right) x\right\|
\end{aligned}
$$

Estimate (6.14) now follows from (6.15), the inequality

$$
\left\|x-\widehat{x}_{N}\right\| \leq\left\|x_{N}-\widehat{x}_{N}\right\|+\left\|x_{N}-x\right\|
$$

and Theorem 6.7.

Now we can state the convergence result for fully discretized collocation method.

Theorem 6.12. Assume that $F(., r) \in\left(\mathcal{C}^{m, \alpha}(0, T]\right)^{2}$ for all $r \in[0,1]$ and $\|F(\cdot, r)\|_{m, \alpha}$ are uniformly bounded with respect to $r$. Assume $K_{+}, K_{-} \in$ $\mathcal{S}^{m, \alpha}$. Let $G$ be the unique solution of the system (3.2). Assume that the fully discretization collocation method (5.10) with collocation parameters $0 \leq$ $c_{1}<\ldots<c_{m} \leq 1, m \in \mathbb{N}$ and with grading parameter $\rho \geq 1$ are used. Then 
there exists an integer $N_{0}$ such that for all $N \geq N_{0}$, operator equation (6.4) possesses a unique solution $\widehat{u}_{N}(., r) \in\left(\mathcal{S}_{m-1}^{(-1)}\left(\triangle_{h}\right)\right)^{2}$ and

$$
\sup _{r \in[0,1]}\left\|G(., r)-\widehat{u}_{N}\right\|_{\infty} \rightarrow 0, \quad N \rightarrow \infty .
$$

Furthermore, for $N \geq N_{0}$ the following error estimates hold:

$$
\sup _{r \in[0,1]}\left\|G(., r)-\widehat{u}_{N}\right\|_{\infty} \leq \operatorname{const} E(N, m, \rho, \alpha) .
$$

Proof. The conditions of Theorem 6.11 are satisfied with $X=\left(\mathcal{L}^{\infty}(0, T)\right)^{2}$, $X^{\prime}=(\mathcal{C}[0, T])^{2}, \mathcal{T}=\mathcal{K}, x=G(., r), \widehat{x}_{N}=\widehat{u}_{N}, p_{N}=\mathbf{p}_{N}$ and $\mathcal{T}_{N}$ the approximation of $\mathcal{K}$ by using the product quadrature rule introduced in Section 5.2. Furthermore the assumption of Theorem 6.11 about $\mathcal{T}_{N}$ is satisfied by iii) of Lemma 6.10. The estimate for the first term in right hand side of (6.14) is given in (6.5). By ii) of Lemma 6.10 we get the estimate of second right hand side term of (6.14). The last term is zero, because in our method $f_{N}=f$.

Similar results also holds when the kernel changes sign on vertical and/or horizontal lines and different graded meshes are used on subintervals where the kernel does not change sign.

6.3. Fuzziness of the approximate solution. The main question is whether the approximate solution is fuzzy. In this section, we propose sufficient conditions which guarantee fuzziness of approximate solution.

Definition 6.13. Suppose $F=[f, \bar{f}]^{T}$ is a vector function. We say $F$ is a strictly fuzzy function if $[\underline{f}, \bar{f}]$ is a fuzzy function and there is $\delta>0$ such that

(1) $\frac{f\left(t, r_{2}\right)-\underline{f}\left(t, r_{1}\right)}{r_{2}-r_{1}}>\delta$ for all $t \in[0, T]$ and $0 \leq r_{1}<r_{2} \leq 1$.

(2) $\frac{\bar{f}\left(t, r_{2}\right)-\bar{f}\left(t, r_{1}\right)}{r_{2}-r_{1}}<-\delta$ for all $t \in[0, T]$ and $0 \leq r_{1}<r_{2} \leq 1$.

(3) $\underline{f}(t, 1)<\bar{f}(t, 1), \quad t \in[0, T]$.

Now, it is possible to prove the fuzziness of $\mathbf{u}_{N}=\left(\underline{u}_{N}, \bar{u}_{N}\right)$ for those $F$ that are strictly fuzzy functions.

In the following theorem by adding some more assumptions on $F$ we guarantee the fuzziness of approximate solution.

Theorem 6.14. Suppose that $F$ is a strictly fuzzy vector function. Let for any $r \in[0,1], F(., r) \in\left(\mathcal{C}^{m, \alpha}(0, T]\right)^{2}$ and $K_{+}, K_{-} \in S^{m, \alpha}$. Then the system (3.2) has a unique solution $G=[\underline{g}, \bar{g}], G(\cdot, r) \in\left(\mathcal{C}^{m, \alpha}(0, T]\right)^{2}$, and $G$ is a strictly fuzzy function. Assume that a collocation method of the form (6.4) with collocation points $0 \leq c_{1}<\ldots<c_{m} \leq 1, m \in \mathbb{N}$ and with grading parameter $\rho \geq 1$ is used. Let $\left\|\frac{F\left(\cdot, r_{2}\right)-F\left(\cdot, r_{1}\right)}{r_{2}-r_{1}}\right\|_{1, \alpha} \leq$ const, where the constant does not depend on $N$ and $r$. Then there exists an integer $N_{0}$ such that for all $N \geq N_{0}$, operator equation (6.4) possesses a unique fuzzy solution $u_{N}$. 
Proof. Fuzziness of the exact solution is proved in Theorem 4.10 [3]. First we prove that if $F$ is a strictly fuzzy function, then the exact solution $G$ is also a strictly fuzzy function. Let $0 \leq r_{1}<r_{2} \leq 1$. Then by the first equation of the system (3.1)

$$
\frac{\underline{g}\left(t, r_{2}\right)-\underline{g}\left(t, r_{1}\right)}{r_{2}-r_{1}}>\frac{\underline{f}\left(t, r_{2}\right)-\underline{f}\left(t, r_{1}\right)}{r_{2}-r_{1}}>\delta .
$$

The second condition of strictly fuzziness follows similarly. To prove the third condition we take $r=1$ in system (3.1) and subtract the two equations.

Next we prove the monotonicity of approximate solution (condition 1 . of Definition 2.1). Let $0 \leq r_{1}<r_{2} \leq 1$. By Theorem 6.8

$$
\begin{aligned}
\left\|\frac{\underline{g}\left(\cdot, r_{2}\right)-\underline{g}\left(\cdot, r_{1}\right)}{r_{2}-r_{1}}-\frac{u_{N}\left(\cdot, r_{2}\right)-\underline{u_{N}}\left(\cdot, r_{1}\right)}{r_{2}-r_{1}}\right\|_{\infty} \\
\quad \leq\left\|\frac{\underline{F}\left(\cdot, r_{2}\right)-\underline{F}\left(\cdot, r_{1}\right)}{r_{2}-r_{1}}\right\|_{1, \alpha} E(N, 1, \rho, \alpha)
\end{aligned}
$$

By assumption, $\left\|\frac{F\left(\cdot, r_{2}\right)-F\left(\cdot, r_{1}\right)}{r_{2}-r_{1}}\right\|_{1, \alpha} \leq$ const, where the constant does not depend on $N$ and $r$. Then for sufficiently large $N$ the right hand side of (6.17) is less than $\delta / 2$ and since $\frac{\underline{g}\left(\cdot, r_{2}\right)-\underline{g}\left(\cdot, r_{1}\right)}{r_{2}-r_{1}}>\delta$, we get $\underline{u_{N}}\left(\cdot, r_{2}\right)-$ $\underline{u_{N}}\left(\cdot, r_{1}\right)>\delta / 2$. Similarly we can prove that $\overline{u_{N}}\left(\cdot, r_{2}\right)-\overline{u_{N}}\left(\cdot, r_{1}\right)<-\delta / 2$.

Similarly we get

$$
\left\|\bar{g}(\cdot, 1)-\underline{g}(\cdot, 1)-\left(\bar{u}_{N}(\cdot, 1)-\underline{u}_{N}(\cdot, 1)\right)\right\| \leq E(N, m, \rho, \alpha),
$$

therefore for sufficiently large $N, \underline{u}_{N}(t, 1)<\bar{u}_{N}(t, 1)$. Hence all conditions of Definition 2.1 are satisfied. In fact we have proved that for $N$ large enough, $\mathbf{u}_{N}$ is a strictly fuzzy function.

Similar result holds also for the fully discretized collocation method.

If $F$ does not satisfy condition 3 of Definition 6.13 , we cannot guarantee that the approximate solution $\mathbf{u}_{N}$ satisfies condition 3 of Definition 2.1. In this case we can modify our approximate solution to make it fuzzy without spoiling the rate of convergence. Assume that for sufficiently large $N$, $\underline{u}_{N}(t, 1)>\bar{u}_{N}(t, 1)$ for some $t \in[0, T]$. Let $r_{N}=\inf \left\{r \in[0,1]: \underline{u}_{N}(t, r)>\right.$ $\left.\bar{u}_{N}(t, r)\right\}$. In this case, we propose to use new forms of approximating functions:

$$
\underline{u}_{N}^{\text {new }}(t, r)= \begin{cases}\underline{u}_{N}(t, r), & \text { if } 0 \leq r<r_{N} \\ \frac{\underline{u}_{N}\left(t, r_{N}\right)+\bar{u}_{N}\left(t, r_{N}\right)}{2}, & \text { if } r_{N} \leq r \leq 1\end{cases}
$$

and

$$
\bar{u}_{N}^{\text {new }}(t, r)= \begin{cases}\bar{u}_{N}(t, r), & \text { if } 0 \leq r<r_{N} \\ \frac{\underline{u}_{N}\left(t, r_{N}\right)+\bar{u}_{N}\left(t, r_{N}\right)}{2}, & \text { if } r_{N} \leq r \leq 1 .\end{cases}
$$

Obviously $\mathbf{u}_{N}^{\text {new }}$ is a fuzzy function. 
Let $t \in[0, T]$ be such that $\underline{u}_{N}(t, 1)>\bar{u}_{N}(t, 1)$ for $N$ large enough and let $r>r_{N}$. Then $\bar{u}_{N}(t, r) \leq \underline{u}_{N}(t, r)$, hence

$$
\bar{u}_{N}(t, r)-\bar{g}(t, r) \leq \bar{u}_{N}(t, r)-\underline{g}(t, r) \leq \underline{u}_{N}(t, r)-\underline{g}(t, r) .
$$

Therefore $\left|\bar{u}_{N}(t, r)-\underline{g}(t, r)\right| \leq \max \left\{\left|\underline{u}_{N}(t, r)-\underline{g}(t, r)\right|,\left|\bar{u}_{N}(t, r)-\bar{g}(t, r)\right|\right\}$. Hence if $N$ is large enough so that the convergence estimate of Theorem 6.8 (or Theorem 6.12) holds, we have for $r \in\left[r_{N}, 1\right]$,

$$
\begin{aligned}
\left|\underline{u}_{N}^{\text {new }}(t, r)-\underline{g}(t, r)\right| & =\left|\frac{\underline{u}_{N}(t, r)+\bar{u}_{N}(t, r)}{2}-\underline{g}(t, r)\right| \\
& \leq\left|\frac{\underline{u}_{N}(t, r)-\underline{g}(t, r)}{2}\right|+\left|\frac{\bar{u}_{N}(t, r)-\underline{g}(t, r)}{2}\right| \leq C E(N, m, \rho, \alpha) .
\end{aligned}
$$

Similar estimate holds also for $\bar{u}_{N}^{\text {new }}(t, r)$ and the proof is also similar. Consequently the convergence estimates also hold for the modified solution.

\section{Numerical EXAMPLES}

In this section, we illustrate the convergence of the fully discretized collocation method by some selected examples In examples 7.1 and 7.2 kernels are non-negative, in example 7.3 the kernel changes the sign. Here we use the following approximations for errors

$$
\underline{E}_{N}=\max _{\eta_{k} \in[0, T]}\left\{\left|\underline{u}_{N}\left(\eta_{k}, r\right)-\underline{g}\left(\eta_{k}, r\right)\right|\right\}
$$

and

$$
\bar{E}_{N}=\max _{\eta_{k} \in[0, T]}\left\{\left|\bar{u}_{N}\left(\eta_{k}, r\right)-\bar{g}\left(\eta_{k}, r\right)\right|\right\},
$$

where $(\underline{g}, \bar{g})$ and $\left(\underline{u}_{N}, \bar{u}_{N}\right)$ (for $N \in \mathbb{N}$ ) are exact and numerical solutions of the system (3.1), respectively, and $\eta_{k}=\frac{k}{10 N}, k=0, \ldots, 10 N$. The approximate order of the convergence can be obtained by using the formula

$$
O_{N}=\log _{2} \frac{E_{N}}{E_{2 N}}
$$

Example 7.1. Consider the system of fuzzy Volterra integral equation with weakly singular kernel (FVIEW) on $[0,1]$ with

$$
\begin{gathered}
K(t, s)=\frac{(t-s)^{2.5}+1}{(t-s)^{0.5}}, \\
\underline{f}(t, r)=\left(t^{0.5}-\frac{\pi}{8} t^{2}-\frac{\pi}{2} t\right)\left(r^{2}+r\right), \\
\bar{f}(t, r)=\left(t^{0.5}-\frac{\pi}{8} t^{2}-\frac{\pi}{2} t\right)\left(4-r^{3}-r\right) .
\end{gathered}
$$

The exact solution is$$
g(t, r)=\left(t^{0.5}\left(r^{2}+r\right), t^{0.5}\left(4-r^{3}-r\right)\right) .
$$

Here $K \in S^{m, 0.5}, f \in \mathcal{C}^{m, 0.5}(0,1]$ and according to Theorem 4.6 in [3], $g \in$ $\mathcal{C}^{m, 0.5}(0,1]$ for any $m \in \mathbb{N}$. We used fully discretized collocation method with 
discontinuous linear splines with two collocation points, and with piecewise constant splines, and a graded mesh with grading parameter $\rho$.

Method (1): $m=2, c_{1}=0.6, c_{2}=0.8$.

Method (2): $m=1, c_{1}=0.4$.

By our convergence results we expect the order of convergence to be $\rho / 2$ for $\rho<2 m$, and $m$ for $\rho \geq 2 m$. In Tables $1-5$ we illustrate the error and order of convergence by applying Method (1), Method (2) on $r=0.9$.

\begin{tabular}{l|cccc}
\hline $\mathrm{N}$ & $\underline{E}_{N}$ & $\bar{E}_{N}$ & $\underline{O}_{N}$ & $\bar{O}_{N}$ \\
\hline 64 & $9.2334 \mathrm{e}-02$ & $1.2803 \mathrm{e}-01$ & & \\
\hline 128 & $6.4451 \mathrm{e}-02$ & $8.9365 \mathrm{e}-02$ & 0.51 & 0.51 \\
\hline 256 & $4.5191 \mathrm{e}-02$ & $6.2659 \mathrm{e}-02$ & 0.51 & 0.51 \\
\hline 512 & $3.1774 \mathrm{e}-02$ & $4.4056 \mathrm{e}-02$ & 0.51 & 0.51 \\
\hline 1024 & $2.2381 \mathrm{e}-02$ & $3.1032 \mathrm{e}-02$ & 0.51 & 0.51
\end{tabular}

TABLE 1. The Errors and orders of Example 7.1 by Method (1) for $\rho=1$.

\begin{tabular}{l|cccc}
\hline $\mathrm{N}$ & $\underline{E}_{N}$ & $\bar{E}_{N}$ & $\underline{O}_{N}$ & $\bar{O}_{N}$ \\
\hline 64 & $1.1140 \mathrm{e}-02$ & $1.5446 \mathrm{e}-02$ & & \\
\hline 128 & $5.5576 \mathrm{e}-03$ & $7.7059 \mathrm{e}-03$ & 1.00 & 1.00 \\
\hline 256 & $2.7758 \mathrm{e}-03$ & $3.8488 \mathrm{e}-03$ & 1.00 & 1.00 \\
\hline 512 & $1.3871 \mathrm{e}-03$ & $1.9233 \mathrm{e}-03$ & 1.00 & 1.00 \\
\hline 1024 & $6.9338 \mathrm{e}-04$ & $9.6141 \mathrm{e}-04$ & 1.00 & 1.00
\end{tabular}

TABLE 2. The Errors and orders of Example 7.1 by Method (1) for $\rho=2$.

\begin{tabular}{l|cccc}
\hline $\mathrm{N}$ & $\underline{E}_{N}$ & $\bar{E}_{N}$ & $\underline{O}_{N}$ & $\bar{O}_{N}$ \\
\hline 64 & $1.5303 \mathrm{e}-02$ & $2.12118 \mathrm{e}-02$ & & \\
\hline 128 & $3.8052 \mathrm{e}-03$ & $5.2760 \mathrm{e}-03$ & 2.00 & 2.00 \\
\hline 256 & $9.4905 \mathrm{e}-04$ & $1.3159 \mathrm{e}-03$ & 2.00 & 2.00 \\
\hline 512 & $2.3718 \mathrm{e}-04$ & $3.2886 \mathrm{e}-04$ & 1.98 & 1.98 \\
\hline 1024 & $6.0028 \mathrm{e}-05$ & $8.3232 \mathrm{e}-05$ & 1.98 & 1.98
\end{tabular}

TABLE 3. The Errors and orders of Example 7.1 by Method (1) for $\rho=4$.

Example 7.2. Consider a FVIEW of the form (1.1) with

$$
K(t, s)=\frac{1}{(t-s)^{0.5}}
$$




\begin{tabular}{l|cccc}
\hline $\mathrm{N}$ & $\underline{E}_{N}$ & $\bar{E}_{N}$ & $\underline{O}_{N}$ & $\bar{O}_{N}$ \\
\hline 64 & $1.5871 \mathrm{e}-01$ & $2.20050 \mathrm{e}-01$ & & \\
\hline 128 & $1.0411 \mathrm{e}-01$ & $1.4435 \mathrm{e}-01$ & 0.60 & 0.60 \\
\hline 256 & $6.8837 \mathrm{e}-02$ & $9.5446 \mathrm{e}-02$ & 0.51 & 0.51 \\
\hline 512 & $3.4095 \mathrm{e}-02$ & $4.7275 \mathrm{e}-05$ & 0.51 & 0.51 \\
\hline 1024 & $1.4045 \mathrm{e}-02$ & $3.3340 \mathrm{e}-02$ & 0.50 & 0.50
\end{tabular}

TABLE 4. The Errors and orders of Example 7.1 by Method

(2) for $\rho=1$.

\begin{tabular}{l|cccc}
\hline $\mathrm{N}$ & $\underline{E}_{N}$ & $\bar{E}_{N}$ & $\underline{O}_{N}$ & $\bar{O}_{N}$ \\
\hline 64 & $1.1202 \mathrm{e}-01$ & $1.5532 \mathrm{e}-01$ & & \\
\hline 128 & $6.2853 \mathrm{e}-02$ & $8.7148 \mathrm{e}-02$ & 0.91 & 0.91 \\
\hline 256 & $3.3659 \mathrm{e}-02$ & $4.6532 \mathrm{e}-02$ & 0.94 & 0.94 \\
\hline 512 & $1.7434 \mathrm{e}-03$ & $2.4173 \mathrm{e}-02$ & 0.97 & 0.97 \\
\hline 1024 & $8.9182 \mathrm{e}-03$ & $1.2365 \mathrm{e}-03$ & 0.98 & 0.98
\end{tabular}

TABLE 5. The Errors and orders of Example 7.1 by Method

(2) for $\rho=2$.

$$
\begin{gathered}
\underline{f}(t, r)=\left(\frac{\sin (t)}{\sqrt{t}}-\pi \sin \left(\frac{t}{2}\right) J_{0}\left(\frac{t}{2}\right)\right)(r), \\
\bar{f}(t, r)=\left(\frac{\sin (t)}{\sqrt{t}}-\pi \sin \left(\frac{t}{2}\right) J_{0}\left(\frac{t}{2}\right)\right)(2-r) .
\end{gathered}
$$

Here $J_{\nu}(z)$ is a Bessel function defined by

$$
J_{\nu}(z)=\left(\frac{z}{2}\right)^{\nu} \sum_{k=0}^{\infty} \frac{\left(-\frac{z}{4}\right)^{k}}{k ! \Gamma(k+v+1)} .
$$

The exact solution of this system is $g(t, r)=(\underline{g}(t, r), \bar{g}(t, r))$ with

$$
\underline{g}(t, r)=\frac{\sin (t)}{\sqrt{t}}(r), \quad \bar{g}(t, r)=\frac{\sin (t)}{\sqrt{t}}(2-r)
$$

Again $K \in S^{m, 0.5}, f \in \mathcal{C}^{m, 0.5}(0,1]$ and $g \in \mathcal{C}^{m, 0.5}(0,1]$ for any $m \in \mathbb{N}$. We used fully discretized collocation method with discontinuous linear splines with different choices of the collocation points, and a graded mesh with grading parameter $\rho$. For this example we use following methods:

Method (1): $m=2$ with $c_{1}=0.5, c_{2}=1$.

Method (2): $m=2$ with $c_{1}=\frac{3-\sqrt{3}}{6}, c_{2}=\frac{3+\sqrt{3}}{6}$ (the roots of shifted Legendre polynomial of degree 2 ).

In Tables $6-11$, we illustrate the error and order of convergence by applying Method (1) and Method (2) on $r=0.9$. Convergence rates are the same when using different collocation points, but the roots of shifted Legendre 
polynomial (Gauss points) give better results, since the approximation of the integrals is better.

\begin{tabular}{l|cccc}
\hline $\mathrm{N}$ & $\underline{E}_{N}$ & $\bar{E}_{N}$ & $\underline{O}_{N}$ & $\bar{O}_{N}$ \\
\hline 64 & $1.9364 \mathrm{e}-02$ & $2.3667 \mathrm{e}-02$ & & \\
\hline 128 & $1.3290 \mathrm{e}-02$ & $1.6244 \mathrm{e}-02$ & 0.54 & 0.54 \\
\hline 256 & $9.2114 \mathrm{e}-03$ & $1.1258 \mathrm{e}-02$ & 0.52 & 0.52 \\
\hline 512 & $6.4252 \mathrm{e}-03$ & $7.8530 \mathrm{e}-03$ & 0.51 & 0.51 \\
\hline 1024 & $4.5008 \mathrm{e}-03$ & $5.5010 \mathrm{e}-03$ & 0.51 & 0.51
\end{tabular}

TABLE 6 . The Errors and orders of Example 7.2 by Method (1) for $\rho=1$.

\begin{tabular}{l|cccc}
\hline $\mathrm{N}$ & $\underline{E}_{N}$ & $\bar{E}_{N}$ & $\underline{O}_{N}$ & $\bar{O}_{N}$ \\
\hline 64 & $2.3215 \mathrm{e}-03$ & $2.8374 \mathrm{e}-03$ & & \\
\hline 128 & $1.1507 \mathrm{e}-03$ & $1.4064 \mathrm{e}-03$ & 1,01 & 1.01 \\
\hline 256 & $5.7291 \mathrm{e}-04$ & $7.0022 \mathrm{e}-04$ & 1.00 & 1.00 \\
\hline 512 & $2.8585 \mathrm{e}-04$ & $3.4937 \mathrm{e}-04$ & 1.00 & 1.00 \\
\hline 1024 & $1.4278 \mathrm{e}-04$ & $1.7450 \mathrm{e}-04$ & 1.00 & 1.00
\end{tabular}

TABLE 7. The Errors and orders of Example 7.2 by Method (1) for $\rho=2$.

\begin{tabular}{l|cccc}
\hline $\mathrm{N}$ & $\underline{E}_{N}$ & $\bar{E}_{N}$ & $\underline{O}_{N}$ & $\bar{O}_{N}$ \\
\hline 64 & $2.6126 \mathrm{e}-03$ & $3.1932 \mathrm{e}-03$ & & \\
\hline 128 & $6.8685 \mathrm{e}-04$ & $8.3948 \mathrm{e}-04$ & 1.92 & 1.92 \\
\hline 256 & $1.7534 \mathrm{e}-04$ & $2.1431 \mathrm{e}-04$ & 1.96 & 1.96 \\
\hline 512 & $4.4197 \mathrm{e}-05$ & $5.4018 \mathrm{e}-05$ & 1.98 & 1.98 \\
\hline 1024 & $1.1079 \mathrm{e}-05$ & $1.3541 \mathrm{e}-05$ & 1.99 & 1.99
\end{tabular}

TABLE 8. The Errors and orders of Example 7.2 by Method (1) for $\rho=4$.

Example 7.3. Let us consider a FVIEW of the form (1.1) on $[0,2]$ with

$$
\begin{gathered}
K(t, s)=\frac{t-1}{(t-s)^{\frac{1}{3}}}, \\
\underline{f}(t, r)=\left(1+t^{4}\right)\left(r^{2}+r\right), \\
\bar{f}(t, r)=\left(1+t^{4}\right)\left(4-r^{3}-r\right) .
\end{gathered}
$$

Here $\alpha=\frac{1}{3}$. This time the kernel changes sign along the line $t=1$. We don't know about exact solution. By Theorem 4.9 in [3] the exact solution belongs to $\left(\mathcal{C}_{d}^{m, \alpha, p}(0, T]\right)^{2}$. In this case we should use graded meshes with different grading parameters on $[0,1]$ and on $[1,2]$. 


\begin{tabular}{l|cccc}
\hline $\mathrm{N}$ & $\underline{E}_{N}$ & $\bar{E}_{N}$ & $\underline{O}_{N}$ & $\bar{O}_{N}$ \\
\hline 64 & $7.6802 \mathrm{e}-03$ & $9.3869 \mathrm{e}-03$ & & \\
\hline 128 & $5.2434 \mathrm{e}-03$ & $6.4086 \mathrm{e}-03$ & 0.55 & 0.55 \\
\hline 256 & $3.6196 \mathrm{e}-03$ & $4.4240 \mathrm{e}-03$ & 0.53 & 0.53 \\
\hline 512 & $2.5173 \mathrm{e}-03$ & $3.0767 \mathrm{e}-03$ & 0.52 & 0.52 \\
\hline 1024 & $1.7595 \mathrm{e}-03$ & $2.1505 \mathrm{e}-03$ & 0.51 & 0.51
\end{tabular}

TABLE 9. The Errors and orders of Example 7.2 by Method (2) for $\rho=1$.

\begin{tabular}{l|cccc}
\hline $\mathrm{N}$ & $\underline{E}_{N}$ & $\bar{E}_{N}$ & $\underline{O}_{N}$ & $\bar{O}_{N}$ \\
\hline 64 & $1.0624 \mathrm{e}-03$ & $1.2985 \mathrm{e}-03$ & & \\
\hline 128 & $5.2787 \mathrm{e}-04$ & $6.4517 \mathrm{e}-04$ & 1.00 & 1.00 \\
\hline 256 & $2.6312 \mathrm{e}-04$ & $3.2159 \mathrm{e}-04$ & 1.00 & 1.00 \\
\hline 512 & $1.3136 \mathrm{e}-04$ & $1.6055 \mathrm{e}-04$ & 1.00 & 1.00 \\
\hline 1024 & $6.5628 \mathrm{e}-05$ & $8.0211 \mathrm{e}-05$ & 1.00 & 1.00
\end{tabular}

TABLE 10. The Errors and orders of Example 7.2 by Method (2) for $\rho=2$.

\begin{tabular}{l|cccc}
\hline $\mathrm{N}$ & $\underline{E}_{N}$ & $\bar{E}_{N}$ & $\underline{O}_{N}$ & $\bar{O}_{N}$ \\
\hline 64 & $2.6425 \mathrm{e}-04$ & $3.2297 \mathrm{e}-04$ & & \\
\hline 128 & $6.2779 \mathrm{e}-05$ & $7.6730 \mathrm{e}-05$ & 2.07 & 2.07 \\
\hline 256 & $1.5120 \mathrm{e}-05$ & $1.8480 \mathrm{e}-05$ & 2.05 & 2.05 \\
\hline 512 & $3.6791 \mathrm{e}-06$ & $4.4966 \mathrm{e}-06$ & 2.03 & 2.03 \\
\hline 1024 & $9.0214 \mathrm{e}-07$ & $1.1026 \mathrm{e}-06$ & 2.07 & 2.07
\end{tabular}

TABLE 11. The Errors and orders of Example 7.2 by Method (2) for $\rho=4$.

We use the fully discretized collocation method with $m=2$ and $c_{1}=0.5$, $c_{2}=1$ for numerical approximation of the solution. The optimal grading parameters which give the convergence order $O\left(h^{2}\right)$, are $\rho=3$ on $[0,1]$ and $\rho=\frac{6}{5}$ on $[1,2]$. Our numerical result should provide fuzzy numbers for every $t$. Since we do not have the exact solution, we use the difference of the approximate solutions with $N$ and $2 N$ as an error estimation. We report the numerical solutions on $r=0.5$ with various values of $N$. In Table 12 the first columns show the estimated errors of the method and the last columns show the order of convergence which is 2 . 


\begin{tabular}{l|cccc}
\hline $\mathrm{N}$ & $\left|\underline{U}_{N}-\underline{U}_{2 N}\right|$ & $\left|\bar{U}_{N}-\bar{U}_{2 N}\right|$ & $\underline{O}_{N}$ & $\bar{O}_{N}$ \\
\hline 32 & - & - & - & - \\
\hline 64 & $3.2350 \mathrm{e}-02$ & $1.4558 \mathrm{e}-01$ & - & - \\
\hline 128 & $8.1722 \mathrm{e}-03$ & $3.6775 \mathrm{e}-02$ & 1.98 & 1.98 \\
\hline 256 & $2.0536 \mathrm{e}-03$ & $9.2411 \mathrm{e}-03$ & 1.99 & 1.99 \\
\hline 512 & $5.1490 \mathrm{e}-04$ & $2.3171 \mathrm{e}-03$ & 1.99 & 1.99 \\
\hline 1024 & $1.2895 \mathrm{e}-04$ & $5.8030 \mathrm{e}-04$ & 1.99 & 1.99
\end{tabular}

TABle 12. The Errors and orders of Example 7.3 for the first and second components.

\section{Conclusion}

In this paper, we investigated a fuzzy Volterra integral equation with a weakly singular kernel. The existence, regularity, and fuzziness of the exact solution have been studied in [3]. Collocation methods on discontinuous piecewise polynomial space are proposed. Convergence analysis and fuzziness of the approximate solution have been considered. Both the analysis and numerical methods show that graded mesh is better than uniform mesh for this problem. The numerical method provided to support the theoretical.

\section{ACKNowledgements}

This work has been supported by the Estonian Research Council Grant PRG864.

Author Contributions. Z. A. and U. K. were involved in the conceptualization, investigation and methodology; Z. A.was involved in writing-original manuscript and experimental part.

\section{DECLARATIONS}

Conflict of interest. The authors declare that they have no conflict of interest.

Human participants or animals performed. This article does not contain any studies with human participants or animals performed by any of the authors.

\section{REFERENCES}

[1] K. Atkinson, W. Han, Theoretical numerical analysis, Springer 39, 2005.

[2] Z. Alijani, U. Kangro, Collocation method for fuzzy Volterra integral equations of the second kind, Mathematical Modelling and Analysis, 25(1)(2020), 146-166.

[3] Z. Alijani, U. Kangro, On the smoothness of solution of fuzzy Volterra integral equation of the second kind with weakly singular kernels, submitted to numerical functional analysis and optimization. 
[4] K. Balachandran and K. Kanagarajan, Existence of solutions of general nonlinear fuzzy Volterra-Fredholm integral equations, International Journal of Stochastic Analysis 2005(3)3 333-343.

[5] B. Bede, Mathematics of Fuzzy Sets and Fuzzy Logic, Springer 2013.

[6] H. Brunner, Volterra integral equations. An introduction to theory and applications. Cambridge Monographs on Applied and Computational Mathematics, Cambridge University Press, 2017.

[7] H. Brunner, Collocation methods for Volterra integral and related functional differential equations, Cambridge University Press, 2004.

[8] H. Brunner, A. Pedas, G. Vainikko, Piecewise polynomial collocation methods for linear Volterra integro-differential equations with weakly singular kernels, SIAM Journal on Numerical Analysis, 39 (2001) 957-982.

[9] P. Diamond, P. Kloeden, Metric topology of fuzzy numbers and fuzzy analysis. In: Dubois, D., Prade, H., et al. (eds.) Handbook Fuzzy Sets Ser., vol. 7, pp. 583-641. Kluwer Academic Publishers, Dordrecht (2000).

[10] P. Diamond, Theory and applications of fuzzy Volterra integral equations, IEEE Transactions on Fuzzy Systems 10(1) (2002) 97-102.

[11] M. Friedman, Ma Ming, A. Kandel, Numerical solution of fuzzy differential and integral equations. Fuzzy Sets and System, 106 (1999) 35-48.

[12] R. Goetschel and W. Vaxman, Elementary fuzzy calculus, Fuzzy Sets and Systems, 18(1986) 31-43.

[13] M. Kolk, A. Pedas, G. Vainikko, High order methods for Volterra integral equations with general weak singularities, Numerical Functional Analysis and Optimization, 30 (2009)1002-1024.

[14] R. Kress, Linear integral equations, Applied Mathematical Sciences, Springer, 2014.

[15] A. Pedas, G. Vainikko, On the regularity of solutions to integral equations with nonsmooth kernel on union of open intervals, Journal of Computational and Applied Mathematics, 229(2009) 440-451.

[16] A. Pedas, G. Vainikko, Superconvergence of piecewise polynomial collocation for nonlinear weakly singular integral equations, Journal of Integral Equations and Applications, 9(1997), 379-406.

[17] F. Saberidad, S. M. Karbassi, M. Heydari, Numerical solution of nonlinear fuzzy Volterra integral equations of the second kind for changing sign kernels. Soft computing, 2018, https://doi.org/10.1007/s00500-018-3668-x.

[18] P. V. Subrahmanyam and S. K. Sudarsanam, A note on fuzzy Volterra integral equations, Fuzzy Sets and Systems 81(2) (1996) 237-240.

[19] G. Vainikko, Multidimensional weakly singular integral equations, Springer, 1993.

[20] G. Vainikko, Weakly singular integral equations, Lecture notes, HUT, UT, 2006-2007, http://kodu.ut.ee/ gen/WSIElecturesSIAM.pdf.

[21] L. A. Zadeh, Fuzzy sets, Information and control, 8 (1965) 338-353.

Institute of Mathematics and Statistics, University of TARtu, Estonia

Email address: zahra.alijani@ut.ee

Institute OF MAThematics AND Statistics, University of TARTU, Estonia

Email address: urve.kangro@ut.ee 